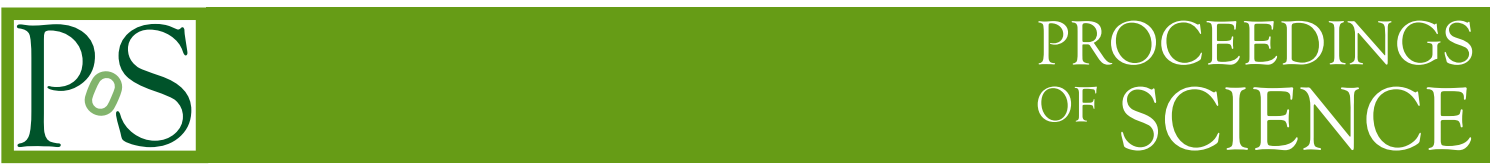

\title{
Multi-Messenger Aspects of Cosmic Neutrinos
}

\author{
Markus Ahlers* \\ WIPAC \& Department of Physics, University of Wisconsin-Madison, Madison, WI 53706, USA \\ E-mail: markus.ahlerseicecube.wisc.edu
}

\begin{abstract}
The recent observation of $\mathrm{TeV}-\mathrm{PeV}$ neutrinos by IceCube has opened a new window to the highenergy Universe. I will discuss this signal in the context of multi-messenger astronomy. For extragalactic source scenarios the corresponding hadronic gamma-rays are not directly observable due to interactions with the cosmic radiation backgrounds. Nevertheless, the isotropic sub-TeV gamma ray background observed by Fermi-LAT contains indirect information from secondary emission produced in electromagnetic cascades. On the other hand, observation of PeV gamma rays would provide a smoking-gun signal for Galactic emission. Interestingly, the overall energy density of the observed neutrino flux is close to a theoretical limit for neutrino production in ultrahigh energy cosmic ray sources and might indicate a common origin of these phenomena. I will highlight various multi-messenger relations and their implications for neutrino source scenarios.
\end{abstract}

The 34th International Cosmic Ray Conference,

30 July- 6 August, 2015

The Hague, The Netherlands

${ }^{*}$ Speaker. 


\section{Introduction}

Modern astronomy comprises a plethora of observational methods designed for the detection of specific cosmic messengers at various energies. Classical photon-based astronomy ranges from low-energy radio observation with arrays of decameter radio dishes to indirect $\mathrm{TeV}$ gamma-ray detection via electro-magnetic showers in our atmosphere. Cosmic rays (CRs) are observed at $\mathrm{GeV}$ energies via direct detection in space-based or balloon-borne particle trackers and indirectly beyond $\mathrm{EeV}$ via extensive air shower arrays covering thousands of square-kilometers on the ground. On the other hand, the data on astrophysical neutrinos have long been limited to the continuous $\mathrm{MeV}$ neutrino emission from nuclear processes in our Sun or the rare $\mathrm{MeV}$ neutrino outburst from the close-by core-collapse supernovae SN 1987A in the Large Magellanic Cloud (for a review see e.g. [1]).

A new window to the high-energy Universe was recently opened by the IceCube observatory with the discovery of a flux of cosmic neutrinos with TeV-PeV energies. Cosmic neutrinos at these energies are expected to be produced by hadronic interactions of high energy CR nucleons with gas (" $p p$ ") and radiation (" $p \gamma$ "). The mesons (mostly pions) produced in these interactions typically carry $20 \%$ of the initial CR nucleon energy and produce a high-energy flux of neutrinos via their decay $\pi^{+} \rightarrow \mu^{+} v_{\mu}$ followed by $\mu^{+} \rightarrow e^{+} v_{e} \bar{v}_{\mu}$, and the charge-conjugate processes. In this decay chain each neutrino receives about $1 / 4$ th of the initial pion energy. The initial ratio of the three neutrino flavors, $v_{e}: v_{\mu}: v_{\tau} \simeq 1: 2: 0$, is then expected to be visible via their oscillation-averaged composition, approximately $v_{e}: v_{\mu}: v_{\tau} \simeq 1: 1: 1$. Neutral pions produced in the same hadronic $\mathrm{CR}$ interactions are expected to produce a high-energy gamma-rays via the decay $\pi^{0} \rightarrow 2 \gamma$.

It is important to emphasize that neutrino emission corresponds to a unique probe of the highenergy Universe. Unlike CRs, neutrinos are not deflected in cosmic magnetic fields over their long propagation distances and unlike gamma-rays they are not absorbed by pair production via $\gamma \gamma$ interactions with radiation in the source or cosmic radiation backgrounds. In the rather wide energy range from about $10 \mathrm{TeV}$ to $10 \mathrm{EeV}$ neutrinos are thus the only pointing probes that can cover cosmic distances. Moreover, unlike gamma rays, the production of high-energy neutrinos requires the presence of CRs and the detection of neutrinos serves as direct probe of the unknown sources of CRs. However, the downside of neutrino astronomy and the reason for the limited data are the many challenges when it comes to their detection.

The outline of this article is as follows. In section 2 we will summarize the recent findings of the IceCube observatory and highlight various interpretations of the signal in terms of Galactic and extragalactic sources. In sections 3 and 4 we will discuss multi-messenger relations between cosmic rays, gamma rays and neutrinos and highlight the implications for various neutrino emission models and future tests. In section 5 we will discuss the prospects of observing individual neutrino sources with present and future observatories before we conclude in section 6 .

\section{Cosmic TeV-PeV Neutrinos}

High-energy neutrinos beyond $\mathrm{TeV}$ interact weakly with matter via deep inelastic scattering. In this process the neutrino flavor eigenstates scatter off quarks via the exchange of $W$ (charged current) and $Z$ (neutral current) bosons. At $\mathrm{PeV}$ energies the mean energy transfer from the neutrino to 
the nucleus reaches its asymptotic level of about $20 \%$ with a total cross section of about $10^{-33} \mathrm{~cm}^{2}$, scaling weakly with energy as $\sigma \propto E_{v}^{0.36}$ [2]. In charged current interactions the neutrino is converted to a high-energy lepton of the corresponding flavor that can be visible in transparent media via Cherenkov light emission.

Due to the rapid decay of the tau and the strong scattering of electrons in the medium the typical signatures of charged current $v_{e}$ and $v_{\tau}$ interactions is in the form of spherical Cherenkov emission ("cascades") that complement the spherical emission from charged particles from the hadronization of the struck nucleus. In the case of neutral current interactions of all neutrino flavors this hadronic cascade is the only part visible in the detector. A special case is the charged current interaction of $v_{\mu}$, where the long-lived secondary muon can be visible as a track. Even a $v_{\mu}$ interacting outside the detector can then be visible if the muon ranges into the instrumented volume. At high energies the muon track follows the initial direction of the neutrino and allows for a good reconstruction of the position of neutrino sources. The large background of atmospheric muons produced in CR showers can be reduced by selecting only up-going tracks; this is the classical neutrino signature at Cherenkov telescopes.

The IceCube observatory located at the geographic South pole is presently the larges neutrino Cherenkov telescope with a fiducial volume of about one cubic-kilometer. The main detector consists of deep Glacial ice that is instrumented with an array of digital optical modules observing the Cherenkov emission of energetic charged particles (see Ref. [3] for details). Recently, the IceCube Collaboration reported the observation of a flux of high-energy neutrinos that was observed in an analysis identifying bright high-energy events with contained interaction vertex, so-called "high-energy starting events" (HESE). A major advancement of the HESE analysis compared to the classical $v_{\mu}$ search is the inclusion of all neutrino flavors and interactions, while atmospheric backgrounds of neutrinos and muons are effectively vetoed. The analysis of three years of IceCube data shows a significance of $5.7 \sigma[4]$.

By now, the IceCube Collaboration has also seen evidence for this astrophysical TeV-PeV neutrino flux via the classical search of up-going tracks initiated by CC $v_{\mu}$ interactions in the vicinity of the detector [5]. A recent combined analysis of IceCube data [6] sensitive to neutrinos in the $10 \mathrm{TeV}$ to $10 \mathrm{PeV}$ energy range indicates a best-fit power law spectrum of

$$
E_{v}^{2} \phi_{v}=(6.7 \pm 1.1) \times 10^{-8}\left(\frac{E_{V}}{100 \mathrm{TeV}}\right)^{0.50 \pm 0.09} \mathrm{GeV} \mathrm{cm}^{-2} \mathrm{~s}^{-1} \mathrm{sr}^{-1}
$$

The observations is consistent with an equal contribution of three neutrino flavors [7, 8, 9].

The most recent update [10] of the HESE analysis including a fourth year of data provides a combined data sample or 54 events, classified as 39 cascades, 14 tracks and 1 coincident (background) event. A subsample of the events with deposited energy (indicated by green numbers) exceeding $60 \mathrm{TeV}$ is shown as the green data points (tracks $\otimes \&$ cascades $\oplus$ ) in Fig. 1. We also show the 21 most energetic events from the analysis of two years of IceCube data [5] as red data points $(\odot)$. The red numbers indicate the most probable neutrino energy if the event has an astrophysical origin following the best-fit spectrum of the analysis. One event indicated $(*)$ coincides with one HESE track event.

The arrival directions of events shown in Fig. 1 are consistent with an isotropic distribution after taking into account the declination dependence of the effective area and Earth absorption. This 


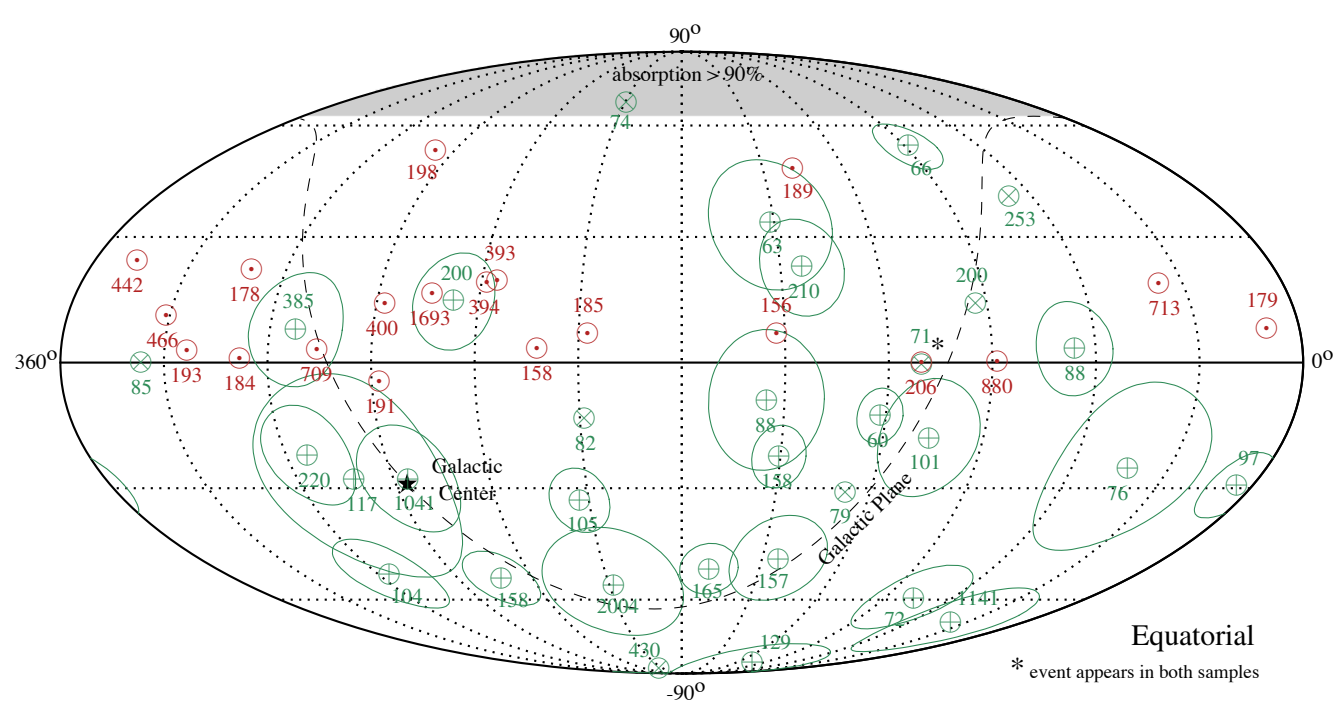

Figure 1: Mollweide projection in equatorial coordinates of the arrival direction of neutrino events. We show the results of the 2-year up-going track analysis [5] with energy proxy MuEx $>50(\odot)$. The red numbers show the most probable neutrino energy (in $\mathrm{TeV}$ ) assuming the best-fit astrophysical flux of the analysis [5]. The events of the 4-year high-energy starting event (HESE) analysis with deposited energy (green numbers) larger than $60 \mathrm{TeV}$ (tracks $\otimes \&$ cascades $\oplus$ ) are also shown [4, 10]. Cascade events $(\oplus$ ) are indicated together with their median angular uncertainty (thin circles). One event $(*)$ appears in both event samples. The grey-shaded region indicate the zenith angle range where Earth absorption of $100 \mathrm{TeV}$ neutrinos is larger than $90 \%$. The dashed line and the star symbol $(\star)$ indicate the Galactic Plane and Center, respectively.

constrains Galactic emission scenarios where one would expect alignment of neutrino arrival directions with Galactic structure. However, the limited event statistics of the samples leave some room for Galactic contributions. Possible Galactic contributions to super-TeV neutrinos are the diffuse neutrino emission of galactic CRs [11, 12, 13], the joint emission of galactic PeV sources [14, 15] or microquasars [16], and extended galactic structures like the Fermi Bubbles [17, 11, 18] or the Galactic halo [19]. A possible association with the sub-TeV diffuse galactic gamma-ray emission [20] and constraints from the non-observation of diffuse galactic PeV gamma-rays [21, 11], have also been investigated. More exotic scenarios have suggested a contribution of neutrino emission from decaying heavy dark matter [22, 23, 24, 25].

However, even with the poor angular resolution of cascade events it is already possible to set limits on Galactic emission. In a recent analysis [26] of the 3-year HESE data with deposited energy of $100 \mathrm{TeV}$ it was argued that diffuse Galactic emission is limited to about $50 \%$ or quasidiffuse emission of Galactic neutrino sources to about $65 \%$. On the other hand, the recent analysis Ref. [27] claims that the 4-year HESE update (see Fig. 1) shows evidence of Galactic emission within latitudes $|b| \leq 10^{\circ}$ above $100 \mathrm{TeV}$. However, the muon neutrino data from the analysis [5] shown as the red data points $(\odot)$ in Fig. 1 do not seem to follow the Galactic Plane in the Northern Hemisphere and challenge this claim.

The absence of significant signs of anisotropy in the data is consistent with an extragalactic 
population of sources. Various extragalactic scenarios have been suggested that might be (partially) responsible for the observed flux of neutrinos. Source candidates include galaxies with intense star formation [28, 29, 30, 31, 32, 33], cores of active galactic nuclei (AGN) [34, 35, 36], low-luminosity AGN [37, 38], blazars [39, 40, 41], low-power GRBs [42, 43, 44], cannonball GRBs [45], intergalactic shocks [46], and active galaxies embedded in structured regions [47, 48, 29].

\section{Neutrinos and Cosmic Rays}

The flux of astrophysical neutrinos is observed between TeV-PeV neutrino energies. In the introduction we have already estimated that hadronic interactions of CRs with gas $(p p)$ or radiation $(p \gamma)$ produce a flux of neutrinos with an energy of about $5 \%$ of the initial CR nucleon. Hence, the CRs responsible for the observed neutrino flux have corresponding energies reaching $20 \mathrm{PeV}$ (proton) to $1 \mathrm{EeV}$ (iron) depending on composition. This composition dependent energy range is above the CR "knee" around $3 \mathrm{PeV}$ and the "ankle" around $3 \mathrm{EeV}$. This region is expected to be the transition region of the spectrum between low-energy Galactic and high-energy extragalactic CRs. It is hence not entirely clear from an energetics point of view if Galactic or extragalactic sources are responsible for the neutrino flux.

Interestingly, the overall energy density of the observed neutrino flux is close to a theoretical limit for neutrino production in the sources of ultra-high energy (UHE) CRs [49]. The neutrino and CR nucleon $(N)$ emission rates $Q$ (in units of $\mathrm{GeV}^{-1} \mathrm{~s}^{-1}$ ) are related via

$$
\frac{1}{3} \sum_{\alpha} E_{v}^{2} Q_{v_{\alpha}}\left(E_{V}\right) \simeq \frac{1}{4} \frac{f_{\pi} K_{\pi}}{1+K_{\pi}} E_{N}^{2} Q_{N}\left(E_{N}\right)
$$

where $f_{\pi} \leq 1$ is the pion production efficiency in hadronic interaction and neutrino and CR nucleon energy are related as $E_{v} \simeq 0.05 E_{N}$. The factor $K_{\pi}$ corresponds to the ratio of charged to neutral pions, which can be approximated as $K_{\pi} \simeq 2$ for $p p$ and $K_{\pi} \simeq 1$ for $p \gamma$ interactions.

After production in hadronic interactions the flux of neutrinos is not further affected by absorption mechanisms and only subject to redshift losses. In the following we assume a flat universe dominated by vacuum energy with $\Omega_{\Lambda} \simeq 0.7$ and cold dark matter with $\Omega_{\mathrm{m}} \simeq 0.3$ [50]. The Hubble parameter at earlier times is then given by its value today of $H_{0} \simeq 70 \mathrm{~km} \mathrm{~s}^{-1} \mathrm{Mpc}^{-1}$ and the relation $H^{2}(z)=H_{0}^{2}\left(\Omega_{\Lambda}+\Omega_{\mathrm{m}}(1+z)^{3}\right)$. The observable diffuse neutrino flux from a homogeneous distribution of extragalactic sources with co-moving density $\mathscr{H}(z)$ can then be readily estimated as

$$
\phi_{v}\left(E_{v}\right)=\frac{1}{4 \pi} \int_{0}^{\infty} \frac{\mathrm{d} z}{H(z)} \mathscr{H}(z) Q_{v}((1+z) E) .
$$

The local emission rate density of UHE CRs depend on spectrum and composition. For an $E^{-2}$ flux of protons it can be estimated as $E_{p}^{2} Q_{p}\left(E_{p}\right) \mathscr{H}(0) \simeq(1-2) \times 10^{44} \mathrm{erg} \mathrm{Mpc}^{-3} \mathrm{yr}^{-1}$ [51]. Hence, using Eq. (3.2) the diffuse neutrino flux can be estimated as

$$
E_{v}^{2} \phi_{v}\left(E_{v}\right) \simeq \frac{\xi_{z} f_{\pi} K_{\pi}}{1+K_{\pi}}(2-4) \times 10^{-8} \mathrm{GeV} \mathrm{cm}^{-2} \mathrm{~s}^{-1} \mathrm{sr},
$$

where we define the evolution factor (assuming constant $f_{\pi}$ )

$$
\xi_{z}=\int_{0}^{\infty} \mathrm{d} z \frac{(1+z)^{-\Gamma}}{\sqrt{\Omega_{\Lambda}+(1+z)^{3} \Omega_{\mathrm{m}}}} \frac{\mathscr{H}(z)}{\mathscr{H}(0)} .
$$


For a spectral index $\Gamma=2$ and no source evolution $(\mathscr{H}(z)=\mathscr{H}(0))$ this evolution factor is $\xi_{z} \simeq 0.5$, whereas evolution following the star-formation rate $[52,53]$ gives $\xi_{z} \simeq 2.4$.

Since the pion production efficiency is limited, $f_{\pi} \leq 1$, the estimate (3.3) provides a theoretical upper limit on neutrino production, the Waxman-Bahcall (WB) bound [49]. It is intriguing that the observed flux (2.1) is very close to this upper limit. This might just be a coincidence, but it can also indicate a multi-messenger relation. It is important to keep in mind that the observed neutrino flux does not directly relate to UHE CRs with energies above $\mathrm{EeV}$ and for proton-dominated scenarios that we assumed in the previous estimate an extrapolation over two orders of magnitude is required.

Neutrino fluxes close to the WB bound would require very efficient CR production with optical thickness $\tau_{p \gamma / p p} \gg 1$, such that $f_{\pi} \simeq 1$, i.e. CR reservoirs [54] such as starburst galaxies [28, 55] or clusters of galaxies $[47,48,56]$. Interestingly, the energy density of Galactic CRs require a similar energy density. Assuming that $1 \%$ of the kinetic energy of $10^{51} \mathrm{erg}$ of a supernova (SN) explosion is converted to CRs and assuming normal galaxies with densities $\mathscr{H}_{0} \simeq 10^{-3} \mathrm{Mpc}^{-3}$ and a SN rate of $10^{-2} \mathrm{yr}^{-1}$ we arrive at $E_{p}^{2} Q_{p}\left(E_{p}\right) \simeq 10^{44} \mathrm{erg} \mathrm{Mpc}^{-3} \mathrm{yr}^{-1}$. This coincidence together with the saturation of the WB bound has let to speculations that Galactic and extragalactic CRs might be produced in the same transient sources [54].

If the sources of UHE CRs are responsible for the neutrino emission it might be possible to cross-correlate these events [57]. The strong interaction of UHE CRs with the cosmic microwave background (CMB) limit the survival length to $200 \mathrm{Mpc}$ above the Greisen-Zatsepin-Kuzmin [58, 59] (GZK) cutoff at around $50 \mathrm{EeV}$. UHE CR sources that appear at larger distance will still be visible by their neutrino emission, but not in UHE CRs. We can make a simple estimate what fraction of neutrinos would be expected to correlate with the local population of UHE CRs which can be sampled by the observed UHE CR arrival direction: The Hubble horizon at about $4.4 \mathrm{Gpc}$ is about 20 times larger than the GZK horizon. That means that we only expect that about one neutrino out of 20 should correlate with local sources. The event sample shown in Fig. 1 corresponds to a total of 73 events with a signal-to-background ratio close to 1 . Hence, we expect only 1-2 of these events to correlate to local UHE CR sources and thus with the arrival direction of observed UHE $\mathrm{CR}$ events. This estimate is consistent with the absence of strong cross-correlations in a dedicated analysis of this data [57].

\section{Neutrinos and Gamma-Rays}

Hadronic interactions of CRs will not only produce charged pions that on decay release a flux of high-energy neutrinos, but also neutral pions that decay via $\pi^{0} \rightarrow 2 \gamma$. The production rates are simply related by

$$
\frac{1}{3} \sum_{\alpha} E_{v}^{2} Q_{v_{\alpha}}\left(E_{v}\right) \simeq \frac{K_{\pi}}{4} E_{\gamma}^{2} Q_{\gamma}\left(E_{\gamma}\right)
$$

where the gamma-ray and neutrino energies are related as $E_{\gamma} \simeq 2 E_{v}$. This relation does not depend on the pion production efficiency and thus the gamma-ray to neutrino multi-messenger relation is less affected by the mechanisms that lead to the initial production of pions, except for the relative charged-to-neutral pion rate $K_{\pi}$ already introduced earlier.

However, the production rate described by Eq. (4.1) is not necessarily the emission rate of the sources. In particular, sources that efficiently produce neutrinos via $p \gamma$ interactions require 


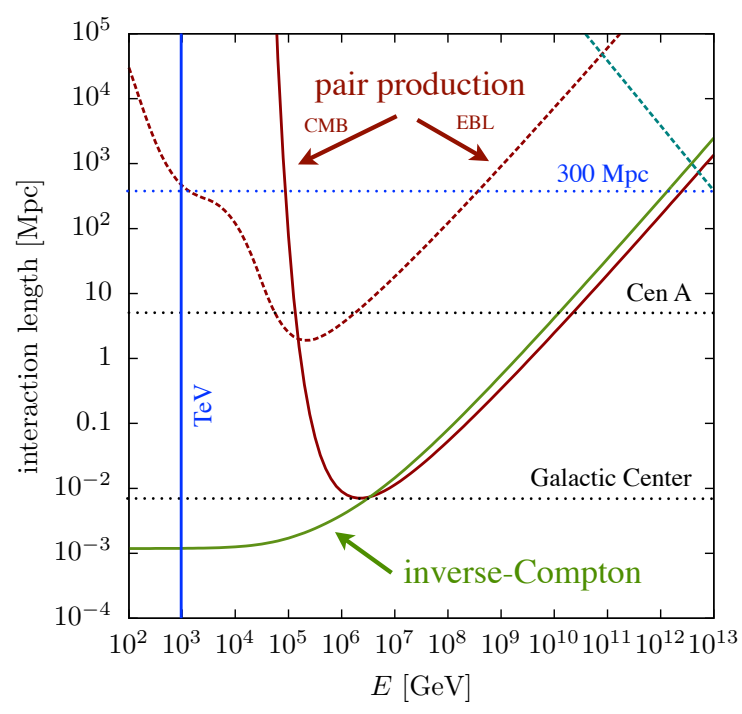

Figure 2: The interaction length of pair production and inverse-Compton scattering of photons with the CMB and EBL. Typical distance scales like the Galactic Center and the close-by radio galaxy Cen A are indicated.

a strong photon target spectrum. The gamma-rays produced via neutral pion decay can in this case undergo $e^{+} e^{-}$pair production before they escape. These leptons can scatter with the same background background photons via inverse-Compton scattering or emit synchrotron emission in magnetic fields. The electromagnetic emission initiated by hadronic interactions might thus look much different from the initial production spectrum (4.1), although the total energy is conserved.

The situation is less complicated for optically thin sources, in particular those sources that are dominated by $p p$ interactions. In this case the hadronic gamma-ray described by Eq. (4.1) are expected to correspond to the source emission spectrum. The pion production efficiency via $p p$ interactions does only weakly depend on the CR energy and the neutrino and gamma-ray spectra are expected to follow the (time-integrated) CR spectrum, $c f$. Eq. (3.1), typically a broken powerlaw up to a maximal energy limited by the CR source type.

However, once released from distant sources it is inevitable that high-energy gamma rays interact with cosmic radiation backgrounds, in particular the CMB. The pair production interaction length of PeV gamma rays in the $\mathrm{CMB}$ is of the order of $10 \mathrm{kpc}$, which makes it impossible to observe this emission over extragalactic distances. Figure 2 shows the interaction length of photons for pair production and inverse-Compton scattering of photons with the CMB and the extragalactic background light (EBL) [60]. Extra-galactic candidate sources for PeV neutrino production, like Centaurus $\mathrm{A}$ at a distance of $4 \mathrm{Mpc}$ shown in the plot, are only visible by hadronic gamma-ray emission below $100 \mathrm{TeV}$. The diffuse flux of gamma-rays from cosmic sources is only visible below $1 \mathrm{TeV}$ due to EBL absorption.

However, pair production and subsequent inverse-Compton scattering of the high energy leptons will lead to electromagnetic cascades that shift the initial radiation into the sub- $\mathrm{TeV}$ gammaray band and supplement the direct sub-TeV emission of the source. The observed gamma-ray background in this energy region provides hence a general upper limit on the diffuse hadronic 

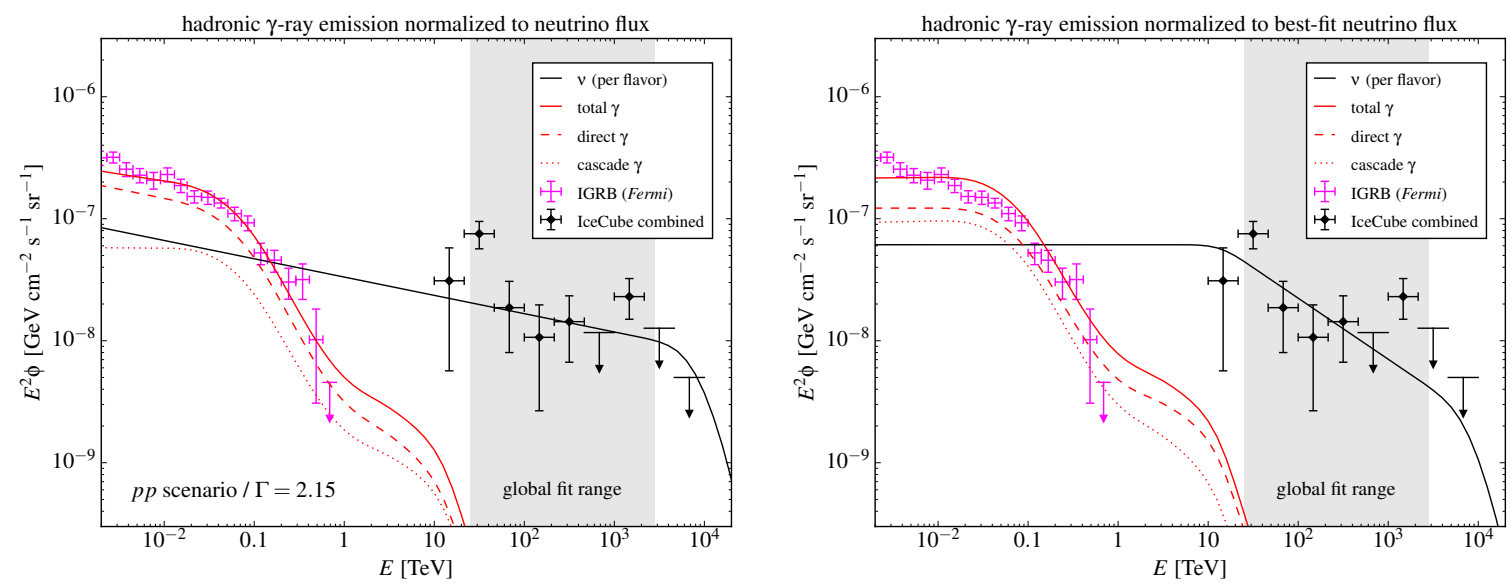

Figure 3: Hadronic gamma-ray (red lines) and neutrino (black line) emission in two $p p$ senarios. The left shows hadronic emission following the relation Eq. (4.1 assuming an unbroken power law with a spectra index $\Gamma=2.15$ and exponential cutoff. The total gamma-ray emission (solid red line) is only marginally consistent with the Fermi IGRB. For illustration we also show the gamma-ray emission decomposed into the direct source emission (dashed red line) and the emission from gamma-ray cascades (dotted red line). The right plot shows a scenario of a broken power-law that reproduces the spectrum in the combined IceCube analysis in the indicated energy region. The turnover to a harder spectrum at lower energy is essential in order to avoid overshooting the Fermi gamma-ray background.

emission [61], which also applies to the production of cosmogenic neutrinos produced via the GZK interaction [62, 63, 64, 65].

Figure 2 shows two diffuse $p p$ emission scenarios that follow the neutrino observation in the $\mathrm{TeV}-\mathrm{PeV}$ energy range. The black and red lines show the resulting neutrino and gamma-ray spectra after accounting for cosmic evolution and cascading in cosmic radiation backgrounds. The left plots shows hadronic emission following the relation Eq. (4.1 assuming a power law $E^{-2.15}$ with an exponential cutoff around PeV, typical for $p p$ scenarios. The gamma-ray spectra is indicated as the sum (solid red line) of direct source emission (dashed red line) and cascaded gamma-rays in the cosmic radiation background (dotted red line). The emission at sub-TeV energies is dominated by the direct photons of the sources. This scenario is marginally consistent with the inferred isotropic diffuse gamma-ray background (IGRB) by Fermi [66]. An unbroken power law emission with a spectral index much softer than $\Gamma \simeq 2.2$ is inconsistent with the IGRB data [29].

The gray shaded area in the plot indicates the fit range of the combined IceCube analysis with best-fit given by Eq. (2.1). Clearly, assuming a soft spectrum with $\Gamma \simeq 2.5$ in this energy range that extends to lower energies will overshoot the gamma-ray background dramatically. However, it is possible in $p p$ scenarios that the $\mathrm{CR}$ spectrum has a harder index at lower energies, e.g. via energy dependent diffusion of CRs out of calorimeters like starburst galaxies [28]. The right plot in Fig. 2 indicates a feasible emission model that reproduces the best-fit neutrino spectrum (2.1) and is marginally consistent with the Fermi IGRB data via a hardening of the emission below $25 \mathrm{TeV}$.

However, a common feature of $p p$ scenarios is the strong contribution of gamma rays in the $10 \mathrm{GeV}$ to $1 \mathrm{TeV}$ band. The extra-galactic gamma-ray background (EGB), including known sources and the IGRB, is expected to be largely dominated by the emission of active galactic nuclei (AGN) at these energies, see e.g. [67, 68]. The contribution of blazars to the Fermi EGB can be studied by 
an analysis of pixel-by-pixel photon count statistics [69]. This places even stronger bounds on $p p$ scenarios that are not expected to coincide with AGN. Recently, it was argued that limits derived from the cross-correlation of Fermi gamma-rays with galaxy catalogue exclude the $p p$ scenarios with soft emission and weak redshift evolution [70]. Other electromagnetic emission bands can also become important in constraining $p p$ emission scenarios, for instance radio emission of galaxy clusters [56].

The situation for $p \gamma$ scenarios is somewhat ifferent. Firstly, the production spectrum of gamma-rays and neutrinos are obtained via a folding of the initial CR spectrum and the photon target spectrum. For typical target spectra present in astrophysical sources these emission spectra are not well described as power laws. Hence, from the spectral fit of the IceCube data in the $\mathrm{TeV}-\mathrm{PeV}$ range we can not make a simple extrapolation towards lower energies. However, even the cascade emission associated with gamma rays emitted in the TeV-PeV is already at a level of $10 \%$ in the $0.1-1 \mathrm{TeV}$ gamma-ray range. Thus, a careful decomposition of the IGRB into contributions of known populations can also constrain this emission scenario.

However, the presence of strong photon target fields in $p \gamma$ sources is also expected to enhance the gamma-ray opacity with respect to pair production. In fact, a high pion production efficiency of more than 0.01 , which can be motivated by the high neutrino intensity at around $10 \mathrm{TeV}$ [71], implies that $p \gamma$ sources are opaque to $1-100 \mathrm{GeV}$ gamma rays. Depending on the low energy tail of the photon target spectrum this opacity can also extend into the TeV-PeV gamma-ray region and hence these sources would not be constrained by either direct observation by Fermi or indirect emission in the IGRB. Neutrinos could be the unique messenger of high-energy processes for these sources.

The arguments in the previous paragraphs focused on the emission of hadronic gamma-rays from extragalactic sources, which are strongly attenuated by the cosmic radiation backgrounds. However, with the limited angular resolution and statistics of the neutrino observation it is possible that extended emission from Galactic sources can contribute to the data. These emission includes the diffuse gamma-ray emission of the Galactic Plane [72], quasi-diffuse emission from the sum of Galactic sources, or extended emission from Fermi Bubbles [73, 74] or Galactic halo [19]. Exotic contributions like decaying heavy dark matter could also produce an extended emission in gammarays and neutrinos [22, 23, 24, 25].

The observation of $\mathrm{PeV}$ gamma-rays with an attenuation length of about $10 \mathrm{kpc}$ via pair production with CMB photons would be a smoking gun for these Galactic production [21, 11]. Various $\mathrm{CR}$ observatories already provide strong TeV-PeV gamma-ray limits, in particular KASCADEGrande [75]. Studies of the isotropic diffuse high-energy gamma-ray emission in the near future with the HAWC observatory [76] and the far future with the air shower arrays LHAASO [77] or HiSCORE [78] can greatly improve the present limits. However, all these experiments are located in the Northern Hemisphere and have only a limited view of the Galactic Center region or the Fermi Bubbles.

\section{Pinpointing Neutrino Sources}

As we discussed in the previous sections, there is mounting evidence that the TeV-PeV flux observed with IceCube follows an isotropic distribution. This would most naturally arise from 
a superposition of faint point-sources of an extra-galactic source population. However, is this consistent with a non-observation of high-energy neutrino point sources so far? For simplicity, let's consider a distribution of continuously emitting sources with the same emission rate $Q_{v}(E) \propto E^{-\Gamma}$ (in units of $\mathrm{GeV}^{-1} \mathrm{~s}^{-1}$ ). As before, the source density or emission is expected to be red-shift dependent with co-moving density $\mathscr{H}(z)$. Then, the individual point-source spectrum $J$ (in units of $\mathrm{GeV}^{-1} \mathrm{~s}^{-1} \mathrm{~cm}^{-2}$ ) at red-shift $z$ can be expressed as

$$
J(z, E)=\frac{(1+z)^{2} Q_{v}((1+z) E)}{4 \pi d_{L}^{2}(z)},
$$

with luminosity distance $d_{L}(z)=(1+z) \int \mathrm{d} z^{\prime} / H\left(z^{\prime}\right)$ defined by the red-shift Hubble expansion rate $H(z)$.

Based on the diffuse flux (2.1) we can then estimate the contribution of individual point sources of the corresponding population [79]. For a continuously emitting source at a distance $d$ the mean neutrino flux is given as

$$
E_{v}^{2} J_{v} \simeq(0.9 \pm 0.3) \times 10^{-12}\left(\frac{\xi_{z}}{2.4}\right)^{-1}\left(\frac{\mathscr{H}(0)}{10^{-5} \mathrm{Mpc}^{-3}}\right)^{-1}\left(\frac{d}{10 \mathrm{Mpc}}\right)^{-2} \mathrm{TeVcm}^{-2} \mathrm{~s}^{-1}
$$

where we used the evolution parameter defined in Eq. (3.4). The similar argument can be made for transient sources [79] with flaring/burst density rate $\mathscr{\mathscr { H }}(z)$. In this case the time-integrated neutrino flux $F$ (in units of $\mathrm{GeV}^{-1} \mathrm{~cm}^{-2}$ ) from an individual transient can be expressed as

$$
E_{v}^{2} F_{v} \simeq(0.3 \pm 0.1)\left(\frac{\xi_{z}}{2.4}\right)^{-1}\left(\frac{\dot{\mathscr{H}}(0)}{10^{-5} \mathrm{Mpc}^{-3} \mathrm{yr}^{-1}}\right)^{-1}\left(\frac{d}{10 \mathrm{Mpc}}\right)^{-2} \mathrm{GeVcm}^{-2}
$$

The two expressions (5.2) and (5.3) are close to the (optimal) sensitivity of IceCube (5-years, $90 \%$ C.L.) to continuous emitters of the order of $E^{2} J_{90} \simeq 10^{-12} \mathrm{TeV} \mathrm{cm}^{-2} \mathrm{~s}^{-1}$ and $E^{2} F_{90} \simeq$ $0.1 \mathrm{GeV} \mathrm{cm}^{-2}$, respectively [80, 81].

Note that the distance $d$ and density $\mathscr{H}$ that appear in Eqs. (5.2) and (5.3) are in general not independent parameters, but are related statistically [79]. The first individual neutrino source of the population will be the brightest one in the field of view (FoV), i.e. the closest one for equalluminosity sources. Figure 2 shows the expected flux range of the closest continuous (top) or transient (bottom) neutrino source assuming a homogeneous local distribution with density $\mathscr{H}_{0}$ or density rate $\dot{\mathscr{H}}_{0}$, respectively (from Ref. [79]). The different shaded bands indicate the $10 \%$ percentiles around the mean (solid line). The calculation assumes a source distribution following that of the star-formation rate. The plots in Fig. 2 also indicate the point-source sensitivity of IceCube in the Northern Hemisphere after five years of observation. IceCube is presently only sensitive to sparse sources with densities of $\mathscr{H}_{0} \lesssim 10^{-7} \mathrm{Mpc}^{-3}$ like flat-spectrum radio quasars or very rare $\dot{\mathscr{H}}_{0} \lesssim 10^{-8} \mathrm{Mpc}^{-3} \mathrm{yr}^{-1}$ transient source classes like gamma-ray bursts.

A higher sensitivity is expected for cross-correlation studies of neutrino events with source catalogues [80, 81]. For instance, a search for the combined neutrino emission from Fermi-observed blazars provided a strong upper limit on the neutrino flux of these sources, that lies below the observed diffuse flux (2.1) [82]. In particular, the sensitivity for neutrino emission coincident with transient sources is much enhanced due to the reduced atmospheric background during a short 

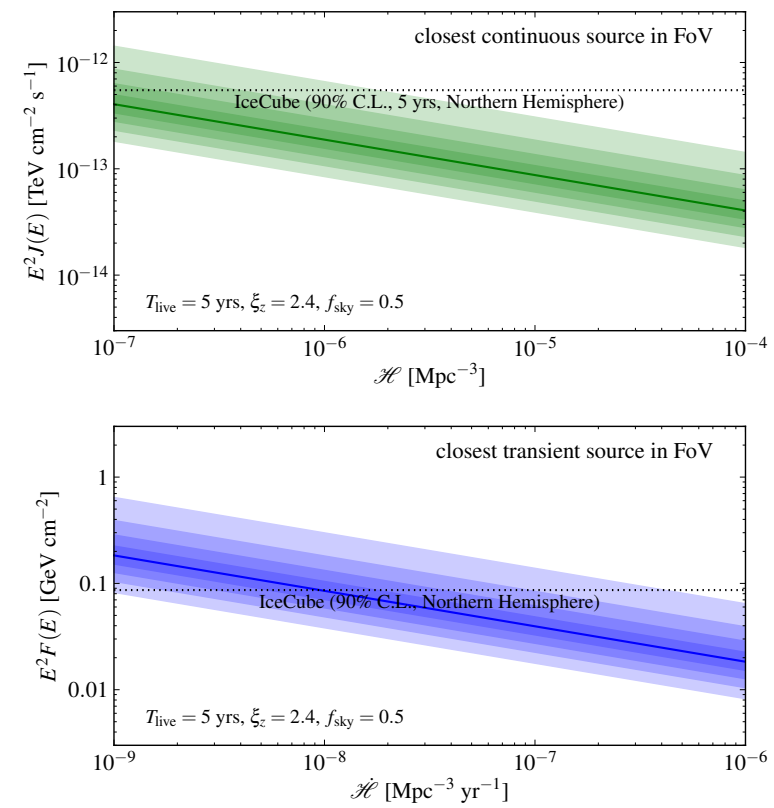

Figure 4: Expected emission of the closest neutrino source in terms of the average source density. The shaded regions show the $10 \%$ percentiles around the mean (solid line) expected from a random distribution of sources (from Ref. [79]).

flaring/burst window [81]. For instance, IceCube has been looking for neutrino emission in coincidence with gamma-ray bursts (GRBs). The present limit on the combined emission from GRBs over a period of five years places an upper limit on their diffuse muon-neutrino flux which is about $1 \%$ of the observed diffuse emission (2.1), constraining the GRB origin of the emission [83].

\section{Conclusions}

The recent observation of TeV-PeV neutrinos has added an important new pillar to multimessenger astronomy. Presently, the observed neutrino events have limited angular resolution and statistics and the underlying source population remains elusive. However, multi-messenger relations between cosmic rays and gamma rays can already provide hints and constraints for possible source scenarios.

One particularly puzzling observation is the particularly high intensity of the IceCube observation in the (10-100) $\mathrm{TeV}$ range, which is indicated by a recent combined data analysis [6]. The underlying source population is required to have a high CR luminosity and pion-production efficiency while at the same time it has to avoid strong limits implied by the observed level of the extragalactic gamma-ray background seen by Fermi.

The continued observation of the neutrino flux with IceCube will help to identify possible substructures in the spectrum that provide indirect evidence of the source emission type. An improved study of neutrino flavor composition will provide an important test of astrophysical neutrino emission and atmospheric background models. Subdominant contributions from Galactic emission could become evident by the manifestation of anisotropies or the detection of hadronic $\mathrm{PeV}$ gamma-ray emission. 
The field of neutrino astronomy would benefit from a large volume neutrino detector like KM3Net located in the Northern Hemisphere for an independent confirmation of the diffuse flux and for point source studies in an extended field of view [84]. Future extensions of the IceCube observatory like IceCube-Gen2 [85, 86] would greatly improve the sensitivity for extra-galactic neutrino sources.

\section{Acknowledgments}

I would like to thank the organizers of the 34th International Cosmic Ray Conference for their invitation. I am grateful for the support of my collaborators, in particular Francis Halzen, Claudio Kopper and Kohta Murase, and the members of the IceCube Collaboration. This work is supported by the U.S. National Science Foundation (NSF) under grants OPP-0236449 and PHY-0236449.

\section{References}

[1] T. K. Gaisser, F. Halzen, and T. Stanev, Particle astrophysics with high-energy neutrinos, Phys. Rept. 258 (1995) 173-236, [hep-ph/ 941038 4]. [Erratum: Phys. Rept.271,355(1996)].

[2] R. Gandhi, C. Quigg, M. H. Reno, and I. Sarcevic, Neutrino interactions at ultrahigh-energies, Phys. Rev. D58 (1998) 093009, [hep-ph/9807264].

[3] C. Kopper, Recent Results in Neutrino Astronomy, in proceedings of The 34th ICRC, 30 July - 6 August, 2015, The Hague, The Netherlands, POS (ICRC2015) XYZ.

[4] IceCube Collaboration, M. Aartsen et al., Observation of High-Energy Astrophysical Neutrinos in Three Years of IceCube Data, Phys.Rev.Lett. 113 (2014) 101101, [arXiv:1405. 5303].

[5] IceCube Collaboration, M. G. Aartsen et al., Evidence for Astrophysical Muon Neutrinos from the Northern Sky with IceCube, Phys. Rev. Lett. 115 (2015), no. 8 081102, [arXiv: 1507.04005 ].

[6] IceCube Collaboration, M. G. Aartsen et al., A combined maximum-likelihood analysis of the high-energy astrophysical neutrino flux measured with IceCube, Astrophys. J. 809 (2015), no. 1 98, [arXiv:1507.03991].

[7] S. Palomares-Ruiz, A. C. Vincent, and O. Mena, Spectral analysis of the high-energy IceCube neutrinos, Phys. Rev. D91 (2015), no. 10 103008, [arXiv: 1502.02649 ].

[8] A. Palladino, G. Pagliaroli, F. Villante, and F. Vissani, What is the Flavor of the Cosmic Neutrinos Seen by IceCube?, Phys.Rev.Lett. 114 (2015), no. 17 171101, [arXiv: 1502.02923 ].

[9] M. Bustamante, J. F. Beacom, and W. Winter, Theoretically palatable flavor combinations of astrophysical neutrinos, arXiv:1506.02645.

[10] C. Kopper, W. Giang, and N. Kurahashi Neilson, Observation of Astrophysical Neutrinos in Four Years of IceCube Data, in proceedings of The 34th ICRC, 30 July - 6 August, 2015, The Hague, The Netherlands, POS (ICRC2015) XYZ.

[11] M. Ahlers and K. Murase, Probing the Galactic Origin of the IceCube Excess with Gamma-Rays, Phys.Rev. D90 (2014) 023010, [arXiv: 1309 . 4077].

[12] J. C. Joshi, W. Winter, and N. Gupta, How Many of the Observed Neutrino Events Can Be Described by Cosmic Ray Interactions in the Milky Way?, Mon. Not. Roy. Astron. Soc. 439 (2014), no. 4 3414-3419, [arXiv:1310 . 5123]. [Erratum: Mon. Not. Roy. Astron. Soc.446,no.1,892(2014)]. 
[13] M. Kachelriess and S. Ostapchenko, Neutrino yield from Galactic cosmic rays, Phys.Rev. D90 (2014) 083002, [arXiv:1405.3797].

[14] D. Fox, K. Kashiyama, and P. Meszaros, Sub-PeV Neutrinos from TeV Unidentified Sources in the Galaxy, Astrophys.J. 774 (2013) 74, [arXiv:1305.6606].

[15] M. Gonzalez-Garcia, F. Halzen, and V. Niro, Reevaluation of the Prospect of Observing Neutrinos from Galactic Sources in the Light of Recent Results in Gamma Ray and Neutrino Astronomy, Astropart.Phys. 57-58 (2014) 39-48, [arXiv:1310 . 7194].

[16] L. A. Anchordoqui, H. Goldberg, T. C. Paul, L. H. M. da Silva, and B. J. Vlcek, Estimating the contribution of Galactic sources to the diffuse neutrino flux, Phys. Rev. D90 (2014), no. 12123010 , [arXiv:1410.0348].

[17] S. Razzaque, The Galactic Center Origin of a Subset of IceCube Neutrino Events, Phys.Rev. D88 (2013) 081302, [arXiv:1309.2756].

[18] C. Lunardini, S. Razzaque, K. T. Theodoseau, and L. Yang, Neutrino Events at IceCube and the Fermi Bubbles, Phys.Rev. D90 (2014) 023016, [arXiv:1311.7188].

[19] A. M. Taylor, S. Gabici, and F. Aharonian, A Galactic Halo Origin of the Neutrinos Detected by IceCube, Phys.Rev. D89 (2014) 103003, [arXiv: 1403.3206$].$

[20] A. Neronov, D. Semikoz, and C. Tchernin, PeV neutrinos from interactions of cosmic rays with the interstellar medium in the Galaxy, Phys.Rev. D89 (2014) 103002, [arXiv:1307.2158].

[21] N. Gupta, Galactic PeV Neutrinos, Astropart.Phys. 48 (2013) 75-77, [arXiv: 1305.4123 ].

[22] B. Feldstein, A. Kusenko, S. Matsumoto, and T. T. Yanagida, Neutrinos at IceCube from Heavy Decaying Dark Matter, Phys.Rev. D88 (2013), no. 1 015004, [arXiv:1303. 7320 ].

[23] A. Esmaili and P. D. Serpico, Are IceCube neutrinos unveiling PeV-scale decaying dark matter?, JCAP 1311 (2013) 054, [arXiv:1308.1105].

[24] Y. Bai, R. Lu, and J. Salvado, Geometric Compatibility of IceCube TeV-PeV Neutrino Excess and its Galactic Dark Matter Origin, arXiv: 1311.5864.

[25] J. F. Cherry, A. Friedland, and I. M. Shoemaker, Neutrino Portal Dark Matter: From Dwarf Galaxies to IceCube, arXiv:1411.1071.

[26] M. Ahlers, Y. Bai, V. Barger, and R. Lu, Galactic TeV-PeV Neutrinos, arXiv: 1505.03156.

[27] A. Neronov and D. V. Semikoz, Evidence for the Galactic contribution to the IceCube astrophysical neutrino flux, arXiv:1509.03522.

[28] A. Loeb and E. Waxman, The Cumulative background of high energy neutrinos from starburst galaxies, JCAP 0605 (2006) 003, [astro-ph/ 0601695 ].

[29] K. Murase, M. Ahlers, and B. C. Lacki, Testing the Hadronuclear Origin of PeV Neutrinos Observed with IceCube, Phys.Rev. D88 (2013), no. 12 121301, [arXiv: 1306.3417 ].

[30] H.-N. He, T. Wang, Y.-Z. Fan, S.-M. Liu, and D.-M. Wei, Diffuse PeV neutrino emission from ultraluminous infrared galaxies, Phys.Rev. D87 (2013), no. 6 063011, [arXiv:1303.1253].

[31] L. A. Anchordoqui, T. C. Paul, L. H. M. da Silva, D. F. Torres, and B. J. Vlcek, What IceCube data tell us about neutrino emission from star-forming galaxies (so far), Phys.Rev. D89 (2014) 127304, [arXiv:1405.7648]. 
[32] X.-C. Chang and X.-Y. Wang, The diffuse gamma-ray flux associated with sub-PeV/PeV neutrinos from starburst galaxies, Astrophys.J. 793 (2014), no. 2 131, [arXiv: 1406.1099 ].

[33] N. Senno, P. Mészáros, K. Murase, P. Baerwald, and M. J. Rees, Extragalactic star-forming galaxies with hypernovae and supernovae as high-energy neutrino and gamma-ray sources: the case of the 10 TeV neutrino data, Astrophys. J. 806 (2015), no. 124, [arXiv: 1501.04934 ].

[34] F. Stecker, C. Done, M. Salamon, and P. Sommers, High-energy neutrinos from active galactic nuclei, Phys.Rev.Lett. 66 (1991) 2697-2700.

[35] F. W. Stecker, PeV neutrinos observed by IceCube from cores of active galactic nuclei, Phys.Rev. D88 (2013), no. 4 047301, [arXiv:1305.7404].

[36] O. Kalashev, D. Semikoz, and I. Tkachev, Neutrinos in IceCube from AGN's, arXiv: 1410.8124.

[37] Y. Bai, A. Barger, V. Barger, R. Lu, A. Peterson, et al., Neutrino Lighthouse at Sagittarius A*, Phys.Rev. D90 (2014) 063012, [arXiv: 1407.2243 ].

[38] S. S. Kimura, K. Murase, and K. Toma, Neutrino and Cosmic-Ray Emission and Cumulative Background from Radiatively Inefficient Accretion Flows in Low-Luminosity Active Galactic Nuclei, Astrophys. J. 806 (2015) 159, [arXiv: 1411.3588 ].

[39] F. Tavecchio and G. Ghisellini, High-energy cosmic neutrinos from spine-sheath BL Lac jets, Mon. Not. Roy. Astron. Soc. 451 (2015), no. 2 1502-1510, [arXiv: 1411.2783 ].

[40] P. Padovani and E. Resconi, Are both BL Lacs and pulsar wind nebulae the astrophysical counterparts of IceCube neutrino events?, Mon.Not.Roy.Astron.Soc. 443 (2014) 474-484, [arXiv: 1406.0376 ].

[41] C. D. Dermer, K. Murase, and Y. Inoue, Photopion Production in Black-Hole Jets and Flat-Spectrum Radio Quasars as PeV Neutrino Sources, JHEAp 3-4 (2014) 29-40, [arXiv: 1406.2633 ].

[42] E. Waxman and J. N. Bahcall, High-energy neutrinos from cosmological gamma-ray burst fireballs, Phys.Rev.Lett. 78 (1997) 2292-2295, [astro-ph/9701231].

[43] K. Murase and K. Ioka, TeV-PeV Neutrinos from Low-Power Gamma-Ray Burst Jets inside Stars, Phys.Rev.Lett. 111 (2013), no. 12 121102, [arXiv:1306.2274].

[44] S. Ando and J. F. Beacom, Revealing the supernova-gamma-ray burst connection with TeV neutrinos, Phys.Rev.Lett. 95 (2005) 061103, [astro-ph/ 0502521 ].

[45] S. Dado and A. Dar, Origin of the High Energy Cosmic Neutrino Background, Phys.Rev.Lett. 113 (2014), no. 19 191102, [arXiv:1405.5487].

[46] K. Kashiyama and P. Meszaros, Galaxy Mergers as a Source of Cosmic Rays, Neutrinos, and Gamma Rays, Astrophys.J. 790 (2014) L14, [arXiv: 1405.3262$].$

[47] V. Berezinsky, P. Blasi, and V. Ptuskin, Clusters of galaxies as a storage room for cosmic rays, Astrophys J. 487 (1997) 529-535, [astro-ph/9609048].

[48] K. Murase, S. Inoue, and S. Nagataki, Cosmic Rays Above the Second Knee from Clusters of Galaxies and Associated High-Energy Neutrino Emission, Astrophys.J. 689 (2008) L105, [arXiv:0805.0104].

[49] E. Waxman and J. N. Bahcall, High-energy neutrinos from astrophysical sources: An Upper bound, Phys.Rev. D59 (1999) 023002, [hep-ph/9807282].

[50] Particle Data Group Collaboration, K. Olive et al., Review of Particle Physics, Chin.Phys. C38 (2014) 090001. 
[51] M. Ahlers and F. Halzen, Minimal Cosmogenic Neutrinos, Phys.Rev. D86 (2012) 083010, [arXiv:1208.4181].

[52] A. M. Hopkins and J. F. Beacom, On the normalisation of the cosmic star formation history, Astrophys.J. 651 (2006) 142-154, [astro-ph/0601463].

[53] H. Yuksel, M. D. Kistler, J. F. Beacom, and A. M. Hopkins, Revealing the High-Redshift Star Formation Rate with Gamma-Ray Bursts, Astrophys.J. 683 (2008) L5-L8, [arXiv: 0804.4008 ].

[54] B. Katz, E. Waxman, T. Thompson, and A. Loeb, The energy production rate density of cosmic rays in the local universe is $\sim 10^{44-45} \mathrm{erg} \mathrm{Mpc}^{-3} \mathrm{yr}^{-1}$ at all particle energies, arXiv:1311.0287.

[55] I. Tamborra, S. Ando, and K. Murase, Star-forming galaxies as the origin of diffuse high-energy backgrounds: Gamma-ray and neutrino connections, and implications for starburst history, JCAP 1409 (2014), no. 09 043, [arXiv: 1404 .1189].

[56] F. Zandanel, I. Tamborra, S. Gabici, and S. Ando, High-energy gamma-ray and neutrino backgrounds from clusters of galaxies and radio constraints, Astron. Astrophys. 578 (2015) A32, [arXiv:1410.8697].

[57] G. Golup, Correlation between the UHECRs measured by the Pierre Auger Observatory and Telescope Array and neutrino candidate events from IceCube, in proceedings of The 34th ICRC, 30 July - 6 August, 2015, The Hague, The Netherlands, POS (ICRC2015) XYZ.

[58] K. Greisen, End to the cosmic ray spectrum?, Phys.Rev.Lett. 16 (1966) 748-750.

[59] G. Zatsepin and V. Kuzmin, Upper limit of the spectrum of cosmic rays, JETP Lett. 4 (1966) 78-80.

[60] A. Franceschini, G. Rodighiero, and M. Vaccari, The extragalactic optical-infrared background radiations, their time evolution and the cosmic photon-photon opacity, Astron.Astrophys. 487 (2008) 837, [arXiv:0805.1841].

[61] V. Berezinsky and A. Y. Smirnov, Astrophysical upper bounds on neutrino-nucleon cross-section at energy $E \geq 3 \times 10^{-17} \mathrm{eV}$, Phys.Lett. B48 (1974) 269-272.

[62] V. Berezinsky and G. Zatsepin, Cosmic rays at ultrahigh-energies (neutrino?), Phys.Lett. B28 (1969) 423-424.

[63] V. Berezinsky, A. Gazizov, M. Kachelriess, and S. Ostapchenko, Restricting UHECRs and cosmogenic neutrinos with Fermi-LAT, Phys.Lett. B695 (2011) 13-18, [arXiv: 1003.1496 ].

[64] M. Ahlers, L. Anchordoqui, M. Gonzalez-Garcia, F. Halzen, and S. Sarkar, GZK Neutrinos after the Fermi-LAT Diffuse Photon Flux Measurement, Astropart.Phys. 34 (2010) 106-115, [arXiv:1005.2620].

[65] G. Decerprit and D. Allard, Constraints on the origin of ultrahigh energy cosmic rays from cosmogenic neutrinos and photons, Astron.Astrophys. 535 (2011) A66, [arXiv:1107.3722].

[66] Fermi Collaboration, M. Ackermann et al., The spectrum of isotropic diffuse gamma-ray emission between $100 \mathrm{MeV}$ and $820 \mathrm{GeV}$, Astrophys. J. 799 (2015), no. 1 86, [arXiv:1410.3696].

[67] M. Ajello et al., The Origin of the Extragalactic Gamma-Ray Background and Implications for Dark-Matter Annihilation, Astrophys. J. 800 (2015), no. 2 L27, [arXiv: 1501.05301$].$

[68] M. Di Mauro, Isotropic diffuse gamma-ray background: unveiling Dark Matter components beyond the contribution of astrophysical sources, in Fifth International Fermi Symposium Nagoya, Japan, October 20-24, 2014, 2015. arXiv: 1502.02566. 
[69] M. Ajello, 2FHL: The second Catalog of Hard Fermi-LAT Sources, in proceedings of The 34th ICRC, 30 July - 6 August, 2015, The Hague, The Netherlands, POS ( ICRC2015) XYZ.

[70] S. Ando, I. Tamborra, and F. Zandanel, Tomographic Constraints on High-Energy Neutrinos of Hadronuclear Origin, arXiv:1509.02444.

[71] K. Murase, D. Guetta, and M. Ahlers, Hidden Cosmic-Ray Accelerators as an Origin of TeV-PeV Cosmic Neutrinos, arXiv:1509.00805.

[72] Fermi Collaboration, M. Ackermann et al., Fermi-LAT Observations of the Diffuse Gamma-Ray Emission: Implications for Cosmic Rays and the Interstellar Medium, Astrophys.J. 750 (2012) 3, [arXiv:1202.4039].

[73] M. Su, T. R. Slatyer, and D. P. Finkbeiner, Giant Gamma-ray Bubbles from Fermi-LAT: AGN Activity or Bipolar Galactic Wind?, Astrophys.J. 724 (2010) 1044-1082, [arXiv: 1005.5480 ].

[74] Fermi Collaboration, M. Ackermann et al., The Spectrum and Morphology of the Fermi Bubbles, Astrophys.J. (2014) [arXiv:1407.7905].

[75] G. Schatz et al., Search for extremely high energy gamma rays with the KASCADE experiment, in in proceedings of The 28th ICRC, pp. 2293-2296, 2003.

[76] HAWC Collaboration, T. DeYoung, The HAWC observatory, Nucl. Instrum. Meth. A692 (2012) $72-76$.

[77] LHAASO, ARGO-YBJ Collaboration, M. Zha, ARGO-YBJ experiment results and prospects in LHAASO project, Int. J. Mod. Phys. Conf. Ser. 10 (2012) 147-158.

[78] M. Tluczykont, D. Horns, D. Hampf, R. Nachtigall, U. Einhaus, M. Kunnas, T. Kneiske, and G. P. Rowell, HiSCORE: A new detector for astroparticle and particle physics beyond 10-TeV, Nucl. Instrum. Meth. A692 (2012) 246-249.

[79] M. Ahlers and F. Halzen, Pinpointing Extragalactic Neutrino Sources in Light of Recent IceCube Observations, Phys. Rev. D90 (2014), no. 4 043005, [arXiv: 1406.2160 ].

[80] IceCube Collaboration, M. G. Aartsen et al., Searches for Extended and Point-like Neutrino Sources with Four Years of IceCube Data, Astrophys. J. 796 (2014), no. 2 109, [arXiv: 1406.6757 ].

[81] IceCube Collaboration, M. Aartsen et al., Search for Time-independent Neutrino Emission from Astrophysical Sources with 3 yr of IceCube Data, Astrophys.J. 779 (2013) 132, [arXiv:1307.6669].

[82] IceCube Collaboration, Glüsenkamp, Th., Analysis of the cumulative neutrino flux from Fermi-LAT blazar populations using 3 years of IceCube data, in 5th Roma International Conference on Astro-Particle physics (RICAP 14) Noto, Sicily, Italy, September 30-October 3, 2014, 2015. arXiv:1502.03104.

[83] IceCube Collaboration, M. Aartsen et al., Search for Prompt Neutrino Emission from Gamma-Ray Bursts with IceCube, arXiv: 1412.6510.

[84] C. James, Highlights from ANTARES, and prospects for KM3NeT, in proceedings of The 34th ICRC, 30 July - 6 August, 2015, The Hague, The Netherlands, POS ( ICRC2015) XYZ.

[85] IceCube Collaboration, M. Aartsen et al., IceCube-Gen2: A Vision for the Future of Neutrino Astronomy in Antarctica, arXiv: 1412.5106.

[86] E. Blaufuss, The IceCube-Gen2 High Energy Array, in proceedings of The 34th ICRC, 30 July - 6 August, 2015, The Hague, The Netherlands, POS ( ICRC2015) XYZ. 